TITLE:

\title{
Level Set-Based Topology Optimization for the Design of an Electromagnetic Cloak With Ferrite Material
}

\section{AUTHOR(S):}

Otomori, Masaki; Yamada, Takayuki; Andkjaer, Jacob; Izui, Kazuhiro; Nishiwaki, Shinji; Kogiso, Nozomu

\section{CITATION:}

Otomori, Masaki ... [et al]. Level Set-Based Topology Optimization for the Design of an Electromagnetic Cloak With Ferrite Material. IEEE Transactions on Magnetics 2013, 49(5): 2081-2084

\section{ISSUE DATE:}

2013-05

URL:

http://hdl.handle.net/2433/175240

\section{RIGHT:}

(c) 2013 IEEE. Personal use of this material is permitted. Permission from IEEE must be obtained for all other uses, in any current or future media, including reprinting/republishing this material for advertising or promotional purposes, creating new collective works, for resale or redistribution to servers or lists, or reuse of any copyrighted component of this work in other works.; この論文は出版社版でありません。引用の際には出版社版をご確認ご利用ください。; This is not the published version. Please cite only the published version. 


\title{
Level Set-Based Topology Optimization for the Design of an Electromagnetic Cloak with Ferrite Material
}

\author{
Masaki Otomori ${ }^{1}$, Takayuki Yamada ${ }^{1}$, Jacob Andkjær $^{2}$, Kazuhiro Izui ${ }^{1}$, Shinji Nishiwaki ${ }^{1}$, and Nozomu Kogiso ${ }^{3}$ \\ ${ }^{1}$ Departmemt of Mechanical Engineering and Science, Kyoto University, Kyoto, Kyoto 606-8501, Japan \\ ${ }^{2}$ Department of Mechanical Engineering, Technical University of Denmark, Lyngby, DK-2800, Denmark \\ ${ }^{3}$ Department of Aerospace Engineering, Osaka Prefecture University, Sakai, Osaka 599-8531, Japan
}

This paper presents a structural optimization method for the design of an electromagnetic cloak made of ferrite material. Ferrite materials exhibit a frequency-dependent degree of permeability, due to a magnetic resonance phenomenon that can be altered by changing the magnitude of an externally applied DC magnetic field. Thus, such ferrite cloaks have the potential to provide novel functions, such as on-off operation in response to on-off application of an external magnetic field. The optimization problems are formulated to minimize the norm of the scattering field from a cylindrical obstacle. A level set-based topology optimization method incorporating a fictitious interface energy is used to find optimized configurations of the ferrite material. The numerical results demonstrate that the optimization successfully found an appropriate ferrite configuration that functions as an electromagnetic cloak.

Index Terms-Electromagnetic cloak, ferrite, level-set method, topology optimization.

\section{INTRODUCTION}

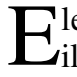
lectromagnetic cloaks are devices that render an object, illuminated by electromagnetic waves of certain wavelengths, undetectable by an observer. The distribution of electric permittivity and magnetic permeability for electromagnetic cylindrical cloaks are theoretically given using transformation optics [1]. However, the obtained theoretical values require that the values change continuously and extremely high values are encountered at the inner radius, so the practical realization of such cloaks is very challenging [2]. On the other hand, design approaches for a cloak made of a simple isotropic material have been reported [3][4], one of which uses a topology optimization method [5], the most flexible type of structural optimization method. Andkjær and Sigmund [3] presented a design method for an optical cloak made of a single-property dielectric material that achieved optical cloaking for waves from certain directions at desirable frequencies, and Andkjær et al. [4] extended this method to a polarization-independent cloak, namely a cloak that functions for both transverse electric (TE) and transverse magnetic (TM) waves.

This study discusses a level set-based topology optimization method for the design of an electromagnetic cloak using a ferrite material. Ferrite materials exhibit a frequencydependent degree of permeability, due to a magnetic resonance phenomenon that can be altered by changing the magnitude of an externally applied DC magnetic field. Thus, such ferrite cloaks have the potential to provide novel functions, such as on-off operation in response to on-off application of an external magnetic field. For the structural design method of an electromagnetic device using a ferrite material, Otomori et al. [6] applied a level set-based topology optimization method incorporating a fictitious interface energy [7] for the design of a metallic waveguide loaded with ferrite. In this study, we extend the previous method, to design an electromagnetic cloak using a ferrite material and air. The extended method deals with both permeability and permittivity distributions, to distinctly represent the ferrite material and air. The objective of the optimization is to find optimized material

Manuscript received October 31, 2012. Corresponding author: Masaki Otomori (e-mail: otomori.masaki.58r@st.kyoto-u.ac.jp).

Digital Object Identifier inserted by IEEE configurations that minimize the norm of the scattering field from a cylindrical obstacle. Therefore, the objective function is set to minimize the norm of the difference between the electric field and a reference field, namely the electric field when there is no scattering object [3][4]. The Landau-Lifshitz model [8] is used to model the permeability of the ferrite material. The optimization algorithm uses the adjoint variable method for the sensitivity analysis and the finite element method (FEM) for solving the electromagnetic propagation and adjoint problems. The 2-dimentional numerical results show that the optimization successfully found an appropriate ferrite configuration that functions as an electromagnetic cloak.

\section{FORMULATION}

\section{A. Ferrite material}

Due to a magnetic resonance phenomenon between the precessional movements caused by electron spin and the impinging microwave radiation, ferrite materials exhibit a frequency-dependent permeability, and their relative permeability can be altered by changing the magnitude of an externally applied DC magnetic field. The magnetic permeability $\bar{\mu}_{f}$ of a ferrite material for a 3-dimensional problem is described using the Landau-Lifshitz model, as follows:

$$
\bar{\mu}_{f}=\left[\begin{array}{ccc}
\mu & \kappa j & 0 \\
-\kappa j & \mu & 0 \\
0 & 0 & 1
\end{array}\right],
$$

where $\mu$ and $\kappa$ in the above equation are given as

$$
\begin{gathered}
\mu=\frac{\omega^{2}-\omega_{0}^{2}}{\omega^{2}-\omega_{h}^{2}}, \\
\kappa=\frac{\omega \omega_{m}}{\omega^{2}-\omega_{h}^{2}},
\end{gathered}
$$

where $\omega$ and $\omega_{0}$ are the angular frequency and precession frequency, respectively. $\omega_{0}, \omega_{h}$, and $\omega_{m}$ are defined as

$$
\begin{gathered}
\omega_{0}=\sqrt{\omega_{h}\left(\omega_{h}+\omega_{m}\right)}, \\
\omega_{h}=\gamma \mu_{0}\left(H_{0}+j \frac{\Delta H}{2}\right), \\
\omega_{m}=\gamma \mu_{0} M_{S},
\end{gathered}
$$




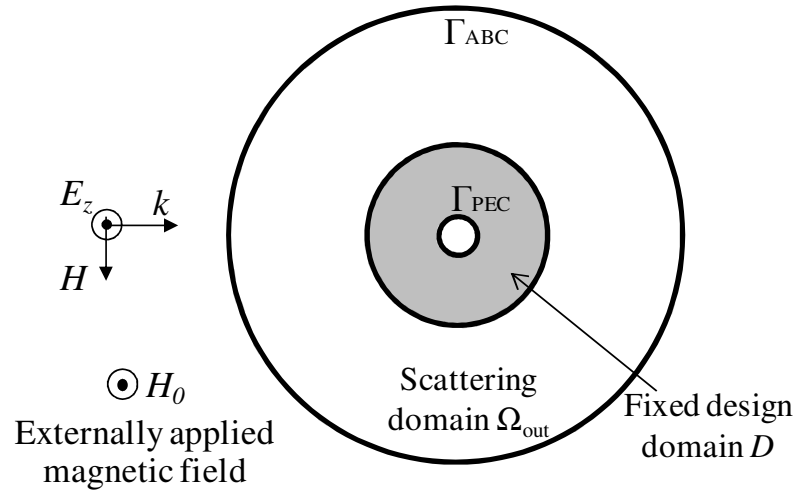

Fig. 1 Analysis domain and boundary conditions for the cylindrical cloak design problem

where $\gamma$ and $\mu_{0}$ are the gyromagnetic ratio of the ferrite and the magnetic permeability of a vacuum, respectively. $H_{0}, \Delta H$, and $M_{S}$ are the magnitude of an externally applied DC magnetic field, the resonance line width of the ferrite material, and the degree of magnetization saturation, respectively.

\section{B. 2 D cloak design problem}

Using the permeability tensor discussed above, the governing equation for a 3-dimensional time harmonic problem using a ferrite material can be described by the following wave propagation equation that is derived from Maxwell's equations.

$$
\nabla \times\left(\bar{\mu}_{f}^{-1} \nabla \times \mathbf{E}\right)-k_{0}^{2} \varepsilon_{f} \mathbf{E}=0,
$$

where $\varepsilon_{f}$ and $k_{0}$ are respectively the relative permittivity of the ferrite and the wave number in a vacuum such that $k_{0}=\omega \sqrt{\varepsilon_{0} \mu_{0}} \cdot \varepsilon_{0}$ and $\mu_{0}$ are respectively the electric permittivity and magnetic permeability in a vacuum, and $\mathbf{E}$ is the electric field. The governing equation for a TE wave propagation problem, where the electromagnetic wave propagates in $x-y$ direction, with the electric field polarized in $z$ direction, is then given by the following Helmholtz equation, derived from Eq.(7), applying the condition that $\partial / \partial z=0$.

$$
\nabla \cdot\left(\mu_{f}^{-1} \nabla E_{z}\right)+k_{0}^{2} \varepsilon_{f} E_{z}=0,
$$

where the relative permeability $\mu_{f}$ of the ferrite for the 2dimensional problem is given as

$$
\mu_{f}=\left[\begin{array}{cc}
\mu & \kappa j \\
-\kappa j & \mu
\end{array}\right] \text {. }
$$

The design domain and boundary conditions of the cloak model are shown in Fig. 1. Incident waves enter the scattering domain from the left, and the outer boundary of this domain is set to function under a first order absorbing boundary condition. The inner boundary is set as a perfect electric conductor (PEC) to represent the scattering object that is to be hidden by the cloak. The governing equation for the cloak design problem is given by

$$
\nabla \cdot\left(\mu_{r}^{-1} \nabla E_{z}\right)+k_{0}^{2} \varepsilon_{r} E_{z}=0,
$$

where $\mu_{r}$ is the relative permeability, $\mu_{r}=\mu_{f}$ in the domain where the ferrite is distributed, and $\mu_{r}=1$ in air.

The boundary conditions are described as follows:

$$
\begin{aligned}
& \mathbf{n} \cdot\left(\mu_{r}^{-1} \nabla \cdot E_{z}\right)+j k_{0} \sqrt{\varepsilon_{r} \mu_{r}^{-1}} E_{z}=j k_{0} \sqrt{\varepsilon_{r} \mu_{r}^{-1}}(1-\mathbf{k} \cdot \mathbf{n}) E_{z}^{\text {inc }} \\
& \text { on } \Gamma_{A B C} \\
& \mathbf{n} \cdot\left(\mu_{r}^{-1} \nabla \cdot E_{z}\right)=0 \begin{array}{ll}
\text { on } \Gamma_{P E C} &
\end{array}
\end{aligned}
$$

where $\mathbf{k}$ is a spatial wave vector, $\mathbf{n}$ is a outward-pointing normal vector, and $E_{z}^{\text {inc }}$ is the incident field.

\section{Optimization Problem}

The purpose of the optimization problem is to find the configuration of ferrite material in the cloak domain that makes the electromagnetic waves propagate as if the scattering object does not exist. Therefore, the objective function is set to minimize the norm of the difference between the electric field and a reference field, namely the electric field when no scattering object is present, as follows [3][4]:

$$
\begin{gathered}
\inf _{\phi} F(\phi)=\int_{\Omega_{\text {out }}}\left(E_{z}-E_{z}^{\mathrm{ref}}\right)^{*}\left(E_{z}-E_{z}^{\mathrm{ref}}\right) \mathrm{d} \Omega, \\
\text { subject to } \text { Helmholtz equation } \\
\text { Boundary conditions }
\end{gathered}
$$

where $E_{z}^{\text {ref }}$ is the reference field and $*$ denotes a complex conjugate. The optimization algorithm uses the adjoint variable method for the sensitivity analysis and the FEM for solving the electromagnetic propagation and adjoint problems.

In the presented method, the relative permeability and permittivity are represented using the characteristic function $\chi_{\phi}$ as follows:

$$
\begin{gathered}
\mu_{r}^{-1}=\left(\mu_{f}^{-1}-\mu_{d}^{-1}\right) \chi_{\phi}+\mu_{d}^{-1} \\
\varepsilon_{r}=\left(\varepsilon_{f}-\varepsilon_{d}\right) \chi_{\phi}+\varepsilon_{d}
\end{gathered}
$$

The characteristic function $\chi_{\phi}$ is defined using the level set function, which is explained in the next subsection. We note that, since the reciprocal form of the relative permeability is included in the governing equation and therefore it is also included in the sensitivity formulation, the reciprocal formulation is used for the permeability representation as Eq.(15), to simplify the derivation of the sensitivity analysis. Further details are provided in [9]. The reciprocal and linear formulations respectively represent the lower and upper theoretical bounds of the effective properties of the composite material [10], so the reciprocal formulation is also physically reasonable.

\section{Level Set-Based Topology Optimization}

Topology optimizations are formulated using a fixed design domain $D$ that consists of a solid domain $\Omega$ filled with material and a void domain $D \backslash \Omega$, with structural boundaries $\partial \Omega$. In a level set-based method, the solid and void domains and the structural boundaries $\partial \Omega$ are expressed using the isosurface of the level set function $\phi$, as follows:

$$
\begin{cases}0<\phi(x) \leq 1 & \text { if } \forall x \in \Omega \backslash \partial \Omega \\ \phi(x)=0 & \text { if } \forall x \in \partial \Omega \\ -1 \leq \phi(x)<0 & \text { if } \forall x \in D \backslash \Omega\end{cases}
$$

The characteristic function $\chi_{\phi}$ that represents the material distribution is described as follows using the level set function defined above:

$$
\chi_{\phi}(\phi)=\left\{\begin{array}{lll}
1 & \text { if } & \phi \geq 0 \\
0 & \text { if } & \phi<0
\end{array}\right.
$$


The optimization problem is then formulated as follows using the above level set-based boundary expressions:

$$
\begin{gathered}
\inf _{\chi_{\phi}} \quad F\left(\chi_{\phi}\right)=\int_{D} f\left(x, \chi_{\phi}\right) d \Omega, \\
\text { s.t } \quad \mathrm{G}\left(\chi_{\phi}\right)=\int_{D} g\left(x, \chi_{\phi}\right) d \Omega-G_{\max } \leq 0,
\end{gathered}
$$

where $f$ is the density function of the objective functional, $g$ is the density function of the constraint functional, and $G_{\max }$ is the upper limit value of the constraint functional.

Because the above defined level set function is allowed to be discontinuous at every point, the optimization problem is an ill-posed problem, so regularization must be applied. In the level set method used here [7], the Tikhonov regularization method is used. A regularization term $\mathrm{R}$ is defined as follows, using a regularization parameter $\tau$,

$$
R=\int_{D} \frac{1}{2} \tau|\nabla \phi|^{2} d \Omega,
$$

and this regularization term is added to the primary objective functional. The above optimization problem is then replaced with the following optimization problem:

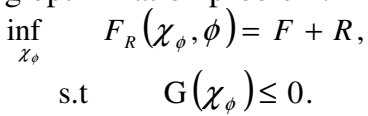

Next, the Karush-Kuhn-Tucker (KKT) conditions of the above optimization problem are derived as follows:

$$
\delta \bar{F}_{R}=0, \lambda G=0, \quad \lambda \geq 0, \quad G \leq 0,
$$

where $\bar{F}_{R}=F_{R}+\lambda G, \lambda$ is the Lagrange multiplier.

Level set functions that satisfy the above KKT conditions can be candidate solutions of the optimization problem, but such solutions are difficult to find directly. Here, we introduce a fictitious time $t$, and assume that the variation of the level set function with respect to time $t$ is proportional to the gradient of Lagrangian $\bar{F}_{R}$, as follows:

$$
\frac{\partial \phi}{\partial t}=-K(\phi) \delta \bar{F}_{R}
$$

where $K(\phi)$ is a coefficient of proportionality.

Substituting Eq.(22) into Eq.(25), and setting an appropriate boundary condition, the following time evolutional equation is obtained.

$$
\left\{\begin{array}{ll}
\frac{\partial \phi}{\partial t}=-K(\phi)\left(\delta \bar{F}-\tau \nabla^{2} \phi\right) & \\
\frac{\partial \phi}{\partial n}=0 & \text { on } \partial D \backslash \partial D_{N} \\
\phi=0 & \text { on } \partial D_{N}
\end{array} .\right.
$$

The optimized problem is then replaced by a problem to solve the above time evolutional equation to update the level set function. We note that the obtained level set function after convergence in the optimization satisfies $\delta \bar{F}_{R}=0$. The constraint functionals are handled using the augmented Lagrangian method [11] by estimating the Lagrange multiplier $\lambda$ at every iteration to satisfy $G(t+\Delta t)=0$. Here, the variation $\delta \bar{F}$ is considered as the topological derivatives [7][12]. Further details are provided in [7].

\section{NUMERICAL EXAMPLES}

Here we apply our method to a cylindrical cloak and a carpet cloak [13] design problem. The conceptual ferrite material used in the two numerical examples has the following

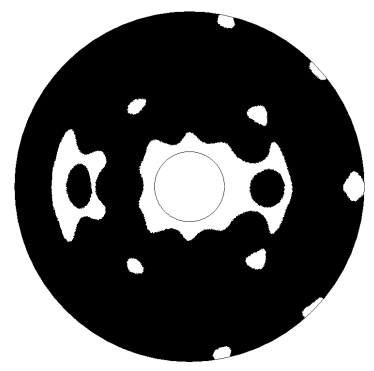

Fig. 2. Optimized material distribution of ferrite material (black) for Example1

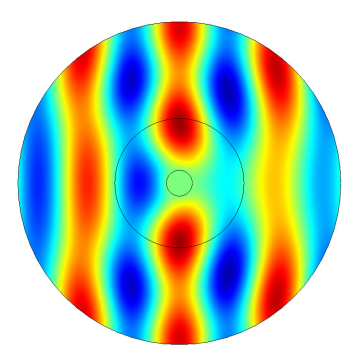

(a)

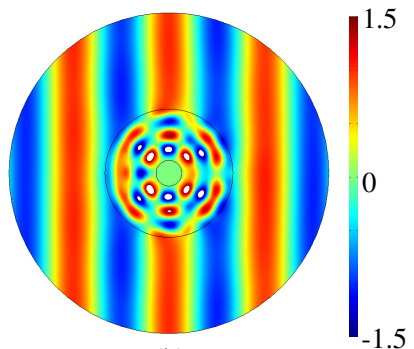

(b)
Fig. 3. Total electric field: (a) no cloak; (b) optimized cloak

parameter values: $\gamma=1.759 \times 10^{11} \mathrm{~T}^{-1} \mathrm{~s}^{-1}, \mu_{0} H_{0}=300 \mathrm{mT}$, $\Delta H=1 \mathrm{mT}$, and $\mu_{0} M_{0}=173 \mathrm{mT}$, assuming that the ferrite material resembles a Yttrium-iron garnet (YIG). The relative permittivity of the ferrite and air are set as $\varepsilon_{f}=10$ and $\varepsilon_{d}=1$, respectively. A configuration filled with ferrite material is used as the initial configuration for both cases. The magnitude of the regularization parameter $\tau$ is set to $1 \times 10^{-4}$.

\section{A. Example 1: Cylindrical cloak design problem}

The design domain and boundary conditions are shown in Fig. 1. The radii of the inner, middle, and outer domains are set to $0.2,1.0$, and $2.5 \mathrm{~m}$, respectively, and the operating frequency is set as $0.2 \mathrm{GHz}$.

Fig. 2 shows the optimized configuration of ferrite material. Fig. 3(a) shows the electric field without the cloak and Fig. 3(b) shows the electric field of the optimized cloak during operation. As Fig. 3(a) shows, the electro-magnetic waves are scattered by the cylindrical object without the cloak, but scattering is much reduced with the cloak operating, as shown in Fig. 3(b). The values of the objective function without and with the cloak are 1.18 and 0.04 , respectively. These results indicate that the optimization successfully found an appropriate ferrite configuration for a cylindrical electromagnetic cloak.

\section{B. Example 2: Carpet cloak design problem}

The design domain and boundary conditions for the carpet cloak are shown in Fig. 4. Here, the inner semi-ellipse located at the bottom center represents the scattering object that is to be hidden by the cloak. The major axis radius of the inner semi-ellipse is set $0.3 \mathrm{~m}$ and the minor axis radius is set to $0.2 \mathrm{~m}$. The major axis radius of the outer semi-ellipse of the cloaking domain is set to $1.0 \mathrm{~m}$ and the minor axis radius is set to $0.5 \mathrm{~m}$. The radius of the outer hemispheres is set to $5.0 \mathrm{~m}$, and the operating frequency is set as $0.5 \mathrm{GHz}$. Here, incident waves enter the scattering domain from the left top, and the electromagnetic waves are reflected at the bottom boundary. The boundary conditions are described as follows: 


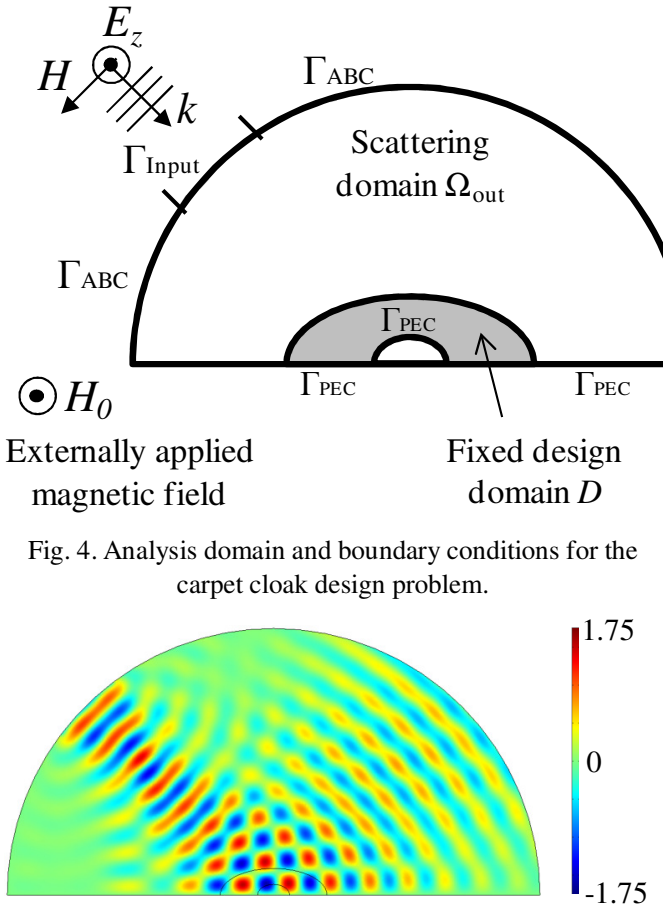

Fig. 5. The reference electric field.

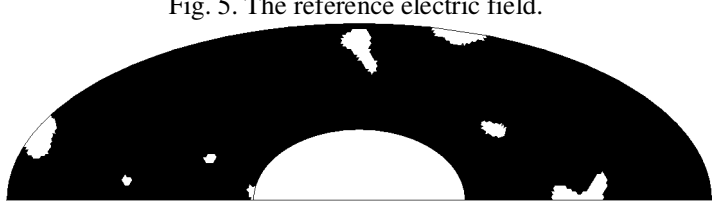

Fig. 6. Optimized material distribution of ferrite material (black) for Example2

$$
\begin{aligned}
& \mathbf{n} \cdot\left(\mu_{r}^{-1} \nabla \cdot E_{z}\right)+j k_{0} \sqrt{\varepsilon_{r} \mu_{r}^{-1}} E_{z}=j k_{0} \sqrt{\varepsilon_{r} \mu_{r}^{-1}}(1-\mathbf{k} \cdot \mathbf{n}) E_{z}^{i n c} \\
& \text { on } \Gamma_{\text {Input }} \\
& \mathbf{n} \cdot\left(\mu_{r}^{-1} \nabla \cdot E_{z}\right)+j k_{0} \sqrt{\varepsilon_{r} \mu_{r}} E_{z}=0 \text { on } \Gamma_{\mathrm{ABC}} \\
& \mathbf{n} \cdot\left(\mu_{r}^{-1} \nabla \cdot E_{z}\right)=0 \text { on } \Gamma_{\mathrm{PEC}}
\end{aligned}
$$

The objective of the optimization is to make the electromagnetic waves reflect as if no scattering object is present at the bottom of the design domain. Fig. 5 shows the reference electric field.

Fig. 6 shows the optimized configuration of ferrite material. Fig. 7(a) shows the electric field without the cloak and Fig. 7(b) shows the electric field of the optimized cloak during operation. As Fig. 7(a) shows, the electromagnetic waves are scattered by the semi-elliptical object without the cloak and they propagate in two directions. However, the scattering is much reduced when the cloak is operating, as shown in Fig. 7(b), where the waves propagate in one direction only. The values of the objective function without and with cloak are 4.46 and 0.24 , respectively. These results demonstrate that the optimization successfully found an appropriate ferrite configuration for a carpet cloak.

\section{CONCLUSION}

In this study, a level set-based topology optimization method incorporating a fictitious interface energy was applied to the design of electromagnetic cloaks using a ferrite material.

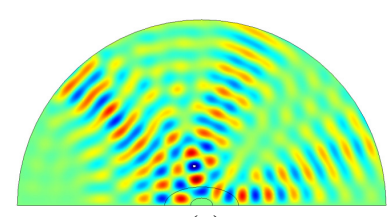

(a)

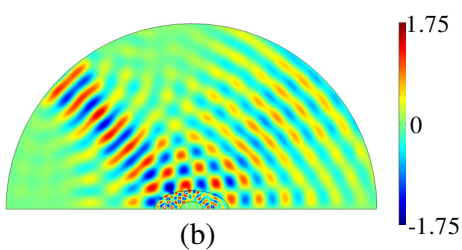

(b)
Fig. 7. Total electric field: (a) no cloak; (b) optimized cloak

The optimization method was formulated to handle the ferrite material and air while considering the relative permeability and permittivity distributions. The objective function was formulated to minimize the norm of the difference between the electric field and a reference field. Numerical examples for two cloak design problems demonstrated that the optimization method successfully found ferrite configurations that function as an electromagnetic cloak. It should be possible to extend the presented method to three-dimensional problems. The current designs are sensitive to the incident wave angle and polarization dependent, and work for a narrow frequency band, but the method for designing cloaks could be improved to handle multi-directional or wide-band cloak designs, by including such requirements into the optimization problems.

\section{ACKNOWLEDGMENT}

This work was partially supported by the JSPS, Grant-inAid for Scientific Research (B), 22360041. The first author is partially supported by AISIN AW CO., LTD. We sincerely appreciate this assistance.

\section{REFERENCES}

[1] J. B. Pendry, D. Schurig and D. R. Smith, "Controlling electromagnetic fields," Science, vol.312, no.5781, pp.1780-1782, 2006.

[2] D. Schurig, J. J. Mock, B. J. Justice, S. A. Cummer, J. B. Pendry, A. F. Starr and D. R. Smith, "Metamaterial electromagnetic cloak at microwave frequencies," Science, vol.314, no.5801, pp.977-980, 2006.

[3] J. Andkjær and O. Sigmund, "Topology optimized low-contrast alldielectric optical cloak," Appl. Phys. Lett., vol.98, no.2, pp.1-3(021112), 2011.

[4] J. Andkjær, N. A. Mortensen and O. Sigmund, "Towards all-dielectric, polarization-independent optical cloaks," Appl. Phys. Lett., vol.100, no.2, pp.1-3(101106), 2012.

[5] M. P. Bendsøe and N. Kikuchi, "Generating optimal topologies in structural design using a homogenization method," Comput. Methods Appl. Mech. Engrg., vol.71, no.2, pp.197-224, Nov. 1988.

[6] M. Otomori, T. Yamada, K. Izui, S. Nishiwaki and N. Kogiso, "Level set-based topology optimization for the design of a ferromagnetic waveguide," IEEE Trans. Magn., vol. 48. no.11, pp. 3072-3075, Nov., 2012.

[7] T. Yamada, K. Izui, S. Nishiwaki and A. Takezawa, "A topology optimization method based on the level set method incorporating a fictitious interface energy," Comput. Method Appl. M., vol. 199, no. 4548, pp. 2876-2891, 2010

[8] B. Lax and K. J. Button, Microwave Ferrites and Ferrimagnetics, New York, McGraw-Hill, 1962.

[9] M. Otomori, T. Yamada, K. Izui, S. Nishiwaki and J. Andkjær, "A topology optimization method based on the level set method for the design of negative permeability dielectric metamaterials," Comput. Method Appl. M., vol. 237-240, pp. 192-221, 2012.

[10] G. W. Milton, The Theory of Composites, Cambridge, Cambridge University Press, 2001.

[11] R.T. Rockafellar, The multiplier method of Hestenes and Powell applied to convex programming, J. Optim. Theory Appl. vol.12, no.6, pp.555$562,1973$.

[12] M. Burger, B. Hackl and W. Ring, "Incorporating topological derivatives into level set methods," J. Comput. Phys., vol.194. no.1, pp. 344-362, 2004

[13] J. Li and J. B. Pendry, "Hiding under the carpet: a new strategy for cloaking,” Phys. Rev. Lett., vol.101. no.20, pp.1-4(203901-1), 2008. 